\title{
Connectivity of Module Device for Small Scale Development
}

\author{
Kosuke Ando ${ }^{a}$, Yuhki Kitazono ${ }^{\text {b,* }}$ \\ National Institute of Technology, Kitakyushu College, 5-20-1 Shii, Kokuraminami-ku, \\ Kitakyushu-city, Fukuoka 802-0985, Japan \\ *Corresponding Author: kitazono@kct.ac.jp
}

\begin{abstract}
Today, we can use modular kit when doing DIY, IoT, robot making easily. It is possible to prepare hardware by merely connecting modularized elements by function. However, the current modular kit has failed to fully demonstrate the function with its connectivity performance due to a bottleneck. We examined the optimum connectivity for the modular kit and verified its cost and performance.
\end{abstract}

Keywords: modular kit, communication, IoT

\section{Introduction}

A modular device is a kit for constructing an ideal system by connecting a device with a certain function and only necessary modules of function. For example, if we want to make a simple robot we will use sensors, actuators and batteries. But if we want to produce a prototype, starting with circuit design is not efficient. By using a modular device, you can configure the system hardware simply by connecting the battery module, sensor module, actuator control module and so on. If you have a module device platform, you can easily write the program. In this way, the modular device is a device that can simply construct the ideal system by connecting only the necessary modules. ${ }^{(1)}$

"MODI" appeared in Cloud Funding as a user friendly modular kit. All modules have the same shape and the same size, and MODI connects with the magnet terminal to configure the hardware. It is affordable and compact size, but because it is a product for IoT and DIY, connectivity of many modules will degrade the performance of the connectivity. It is difficult to use for prototype development etc. ${ }^{(2)}$

First of all, we surveyed what type of scene this kind of modular kit is developed for. Then, it was found that the purpose of the modular kit was narrowed down for each
Table 1. Comparison of communication standard.

\begin{tabular}{|c|c|c|c|c|}
\hline & USB & Ethernet & PCle & I2C/SPI \\
\hline PnP & $\checkmark$ & $\checkmark$ & & \\
\hline Speed & $12[\mathrm{Mbps}]$ & $10[\mathrm{Mbps}]$ & $500[\mathrm{Mbps}]$ & \\
& $480[\mathrm{Mbps}]$ & $100[\mathrm{Mbps}]$ & $\sim$ & $\sim 100[\mathrm{Mbps}]$ \\
& $5[\mathrm{Gbps}]$ & $1000[\mathrm{Mbps}]$ & $64[\mathrm{Gbps}]$ & \\
\hline Max & 127 & No limit & 1 & No limit \\
\hline Cost & $\sim \$ 1$ & $\sim \$ 5$ & & \\
& $\sim \$ 3$ & $\sim \$ 10$ & $\$ 50 \sim$ & $\sim \$ 5$ \\
& $\sim \$ 8$ & $\sim \$ 20$ & & \\
\hline
\end{tabular}

product, such as for robotics, IoT, prototype.

We investigated the proper connectivity to develop a modular kit that is inexpensive and capable of meeting all needs.

\section{Configuration}

\subsection{A selection of communication standard}

Initially we chose several candidates as communication standards for modular devices. For this time, we selected four communication standards, USB, Ethernet, PCIe, I2C / SPI.

We examined the simple characteristics of each of these candidates. Features required for modular devices are as follows

- Whether it is Plug and Play

- communication speed

- Maximum number of connectable units - price

First, the Plug and Play function means that you can join the network automatically when you add a node into the running network. Automatic participation in the network is important in the modular kit.

The second is the evaluation of the communication 


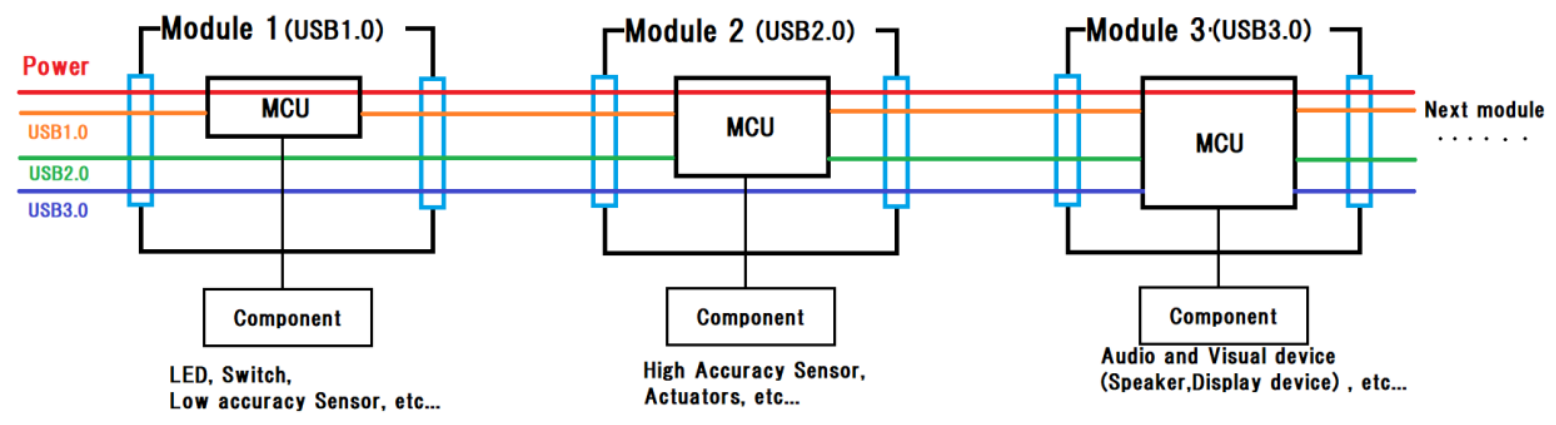

Fig. 1. Module configuration image.

speed. The MODI mentioned above was a bottleneck in communication speed and was not suitable for large scale. To solve this problem, a large communication band is required.

The third is the maximum number of connected units. Some communication standards limit the maximum number of connected devices. This is because it is a standard assuming 1: 1 communication, or because it is the limit of the communication band even assuming $n$ : $n$ communication.

Finally, it is about price. Because we aim to be a device that can be used from simple DIY and IoT to

prototype development, it is not possible to spend the communication standard itself.

Figure 1 shows the survey of these four items. ${ }^{(3)(4)(5)}$ It is the maximum condition in the modular kit that it is Plug and Play and that the maximum number of connected units is large. ${ }^{(6)}$ Furthermore, considering that cost performance is high with respect to the communication band, we thought that it is most appropriate to use the USB standard in the modular kit.

\subsection{Suitable circuit depends on module's value}

We thought about adopting USB as a modular device, but USB has standards of all versions. USB has mainly USB 1.0, USB 2.0, USB 3.0, USB 3.1. There are differences in the communication speed, the number of circuits, and the number of communication buses. Also, as there is a difference in cost of adoption, it is not possible to install USB 3.1, which enables the highest speed communication for all modules.

Therefore, we consider changing the communication circuit to be installed according to the bandwidth that the module would need. For example, in DIY and IoT, the difference between the communication band to be used will be different between the sensor with low precision and the sensor with high institution. Here, the USB2.0 circuit is used for low accuracy sensors, and the USB 3.0 circuit is installed for sensors with high accuracy. By doing this, it is possible to realize a module kit that keeps compatibility and does not put pressure on communication bandwidth.

\subsection{Module connection image}

The connection image of the module is shown in Figure 1. We assume that the module is equipped with two connectors and that the MPU is built in. Components are connected to the MPU. To accommodate IoT to prototype development, the components connected to the module range from LED, switch, sensor to actuator, audio and display.

Module 1 is equipped with a USB 1.0 compatible MCU and a USB controller. As USB 1.0 is slow at the maximum speed of $12 \mathrm{Mbps}$, connect components that are not required to be responsive, such as LEDs, switches, and low-precision sensors. The USB 2.0 and USB 3.0 buses do not pass through the MPU within this module. Module 2 has built-in USB 2.0 compatible MCU and USB controller. USB 2.0 has a maximum speed of 480 [Mbps] and connects components such as high precision sensors and actuators. The USB 3.0 bus does not pass through the MPU within this module. Module 3 has built-in USB 3.0 compatible MCU and USB controller. Because USB 3.0 has a maximum speed as fast as 5 [Gbps], connect an audiovisual device that is required for quick response.

\subsection{Hardware structure}

Different circuits are used for Module 1/2 and Module 3 in Fig. 1 respectively. The circuit configuration pattern is 
shown in Figure 2. A microcontroller is installed in the USB 1.0 and USB 2.0 modules. This is because Module 1/2 is not equipped with components that require quick response. On the other hand, Module 3 is equipped with FPGA and USB 3.0 FIFO Controller. If serial communication is used for USB 3.0 control, that serial communication becomes a bottleneck and USB communication becomes slow. Therefore, using the control method called asynchronous FIFO eliminates the bottleneck between the CPU and the USB 3.0 controller.

It is thought that Module $1 / 2$ does not change the communication speed according to the performance of Microcontroller, but in Module 3 the change will be great depending on FPGA performance. For that reason, the module developer will select the FPGA according to the desired communication band, so the price of the module will depend on the performance.

\section{Experiment}

Next, we measured the data transfer rate using FPGA and USB 3.0 FIFO Controller. We used the FT 601 Q board as USB 3.0 FIFO Controller. In addition, we used Spartan-3E (XC 3 S250 E - 4 VQG 100 C) as the FPGA. ${ }^{(7)}$

In verification, we assumed that a visualizer was created using a modular kit, we sent image data to the display module in a single direction. Uncompressed Full-HD, 60 fps images use the communication bandwidth of $1920 * 1080 * 24 * 60=2847$ [Mbps], but the USB 3.0 FIFO bus speed is 314 [Mbps] = 2512 [Mbps] did. The result of actual measurement is shown in Fig 3.

In this experiment, measurement was performed only with reading, and the size of one packet was 16777216 [Byte], and the size of Queue was 16 [Byte].

Also, we investigated how much the cost of each USB1.0, USB2.0, USB3.0 module circuit would be. The following is an approximate price prediction.

USB 1.0: $\$ 5$

USB 2.0: $\$ 8$

USB 3.0 (FIFO, comm bandwidth = $2847[\mathrm{Mbps}])$ : $\$ 30$

Most of the price of USB 3.0 is FPGA.

\section{Conclusions}

In this paper, in order to develop a modular kit that can be utilized in every situation, we considered new connectivity of modular devices. Also, it was verified to
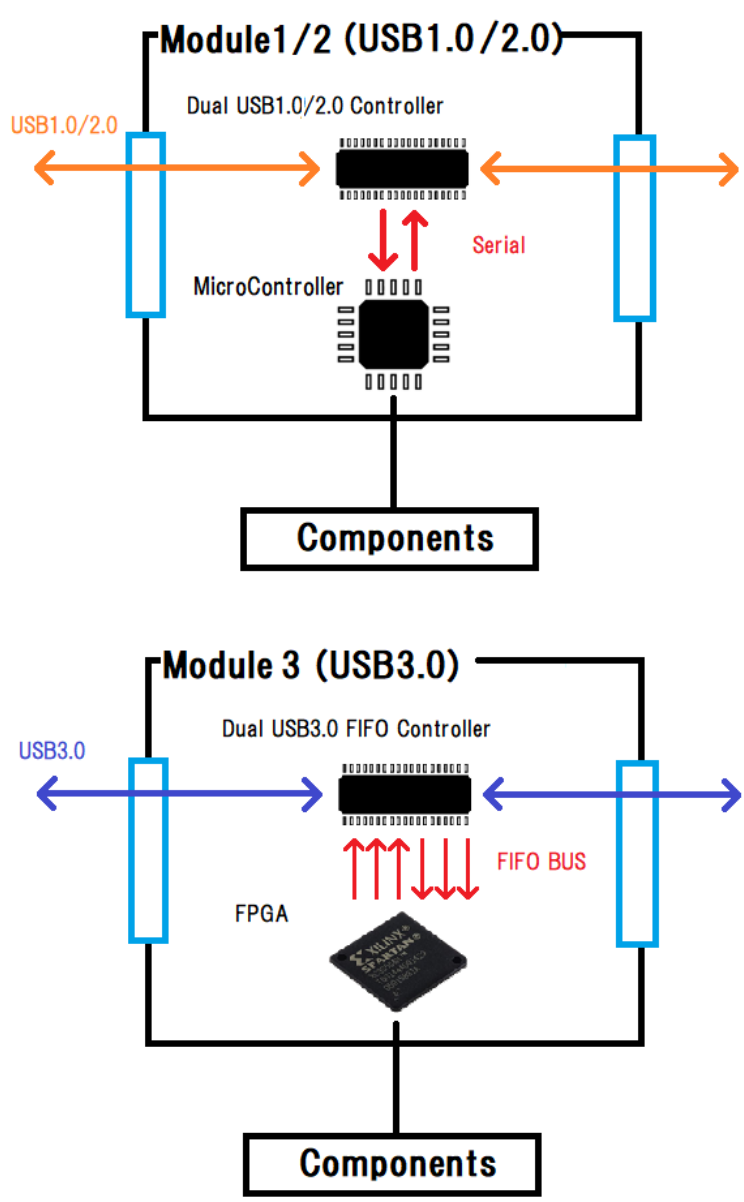

Fig. 2. Circuit configuration diagram

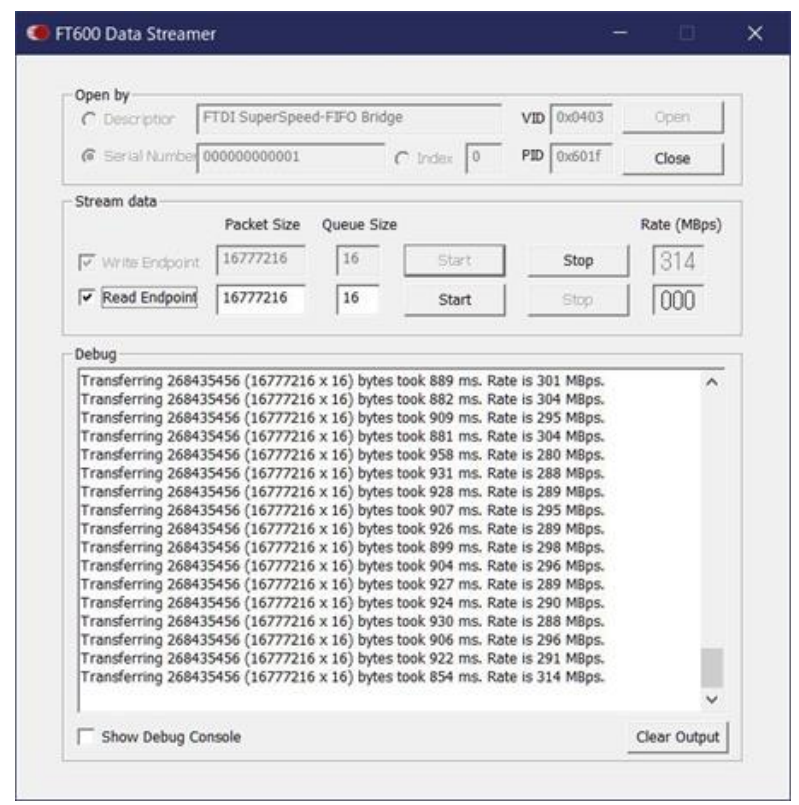

Fig. 3. USB3.0 FIFO real data rate measurement 
what extent bandwidth could be handled actually. The modular kit has lower utility value if its body is expensive relative to the Component. Therefore, we think that if the price of the module changes with respect to the value of the Component, the value of use increases.

\section{References}

(1) https://www.littlebits-jp.com/

(2) KICKSTARTER, "MODI: Create Anything You Want with Robotics of Things.", https://www.kickstarter.com/projects/luxrobo/modi-cre ate-anything-you-want-with-robotics-of-thi?lang=ja

(3) IEEE Xplore Digital Library, 802.3-2015- IEEE Standard for Ethernet,

https://ieeexplore.ieee.org/document/7428776/

(4) Universal Serial Bus

http://www.usb.org/developers/docs/

(5) PCI Express

https://en.wikipedia.org/wiki/PCI_Express

(6) International Organization for Standardization, https://www.iso.org/news/2012/01/Ref1500.html

(7) XILINX, Spartan-3E FPGA Family Data Sheet Production Specification, https://www.xilinx.com/support/documentation/data_sh eets/ds312.pdf 